\title{
Bioink of Multi-Biomaterials with Tyrosine Reinforcement for 3D Bioprinting of Skin Constructs
}

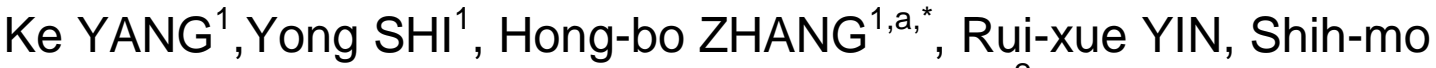 \\ YANG and Wen-jun ZHANG ${ }^{2}$ \\ ${ }^{1}$ Complex and intelligent research center, East China University of Science and \\ Technology, Shanghai, P. R. China, 200237 \\ ${ }^{2}$ Department of Mechanical Engineering, University of Saskatchewan, Canada \\ ahbzhang@ecust.edu.cn \\ ${ }^{*}$ Corresponding author
}

Keywords: Bioink, 3D bioprinting, Tyrosine, Skin scaffolds.

\begin{abstract}
In this paper, a bioink composed of multiple native materials with tyrosine micro-particle reinforcement for 3D bioprinting of skin scaffolds was developed. The rheological property of the developed bioink was optimized for 3D Bioprinting process. The results showed that Tyrosine micro-particle reinforcement strongly enhanced the mechanical strength. Keratinocyte cell culture showed that the 3D bioprinted skin substitutes made of multi-materials bioink is promising in skin repair.
\end{abstract}

\section{Introduction}

Tissue-engineered skin substitutes are 3D structures made of biomaterials that support cell attachment and growth, at the meantime degrade while cells grow [1]. The substitutes then gradually become incorporated in the wound site. More recent advances in tissue engineered skin substitutes involve scaffolds with pre-population of living cells of autologous or allogeneic origin, usually keratinocytes or fibroblasts [2-4].

$3 \mathrm{D}$ bioprinting has shown great success in skin tissue engineering $[5,6,7]$. In 3D printing process bioinks are used to deliver cells and support cell growth, also to provide structural integrity for cells and ability to access essentials while differentiate and proliferate.

Hydrogels are commonly used bioinks, however hydrogels are weak in nature and bioprinting of 3D constructs with complex and intricate micro-architectures still remains a huge challenge[8]. The common approach to address this issue involves the use of highly concentrated polymer solutions, which imposes severe restrictions to the cell mobility within the polymer network and might ultimately lead to poor cell adhesion, and even cell death [9].Thus, further improvement of bioinks for 3D bioprinting is urgently needed. There are several methods have been utilized to achieve better stiffness, such as combination of different materials, incorporation with inorganic particles, fibers, multiple cross linking methods, or with supporting (reinforcement) structures[10-13]. However, none of these bioinks were designed with specific requirement for skin substitutes. Tyrosine is an essential element in melanogenesis, which regulates the microenvironment in skin repair and it is also used as drug delivering vehicles to enhance skin permeability [14, 15]. 
In this paper, bioinks made of Chitosan, Collagen and Hyaluronic with Tyrosine particles reinforcement were developed. Tyrosine particle reinforcement combined with multi-natural biomaterials are two strategies to enhance the mechanical strength and biocompatibility of the bioinks.

\section{Materials and Methods}

Chitosan (Chs, D.D=80\%-90\%) and Type I collagen (Col) were purchased from Zhejiang Golden Shell Pharmaceutical Co. Ltd. and Sigma C-9879, respectively. Hyaluronic acid (HA, $\mathrm{M}_{\mathrm{w}}=10000$ ) was supplied by Bloomage Freda Biopharm Co. Ltd. Tyrosine $\left(\mathrm{M}_{\mathrm{w}}=181.9\right)$ was purchased from Baomanbio. Sodium Tripolyphosphate (STPP, $\mathrm{M}_{\mathrm{w}}=367.86$ ) was purchased from Shanghai Macklin Biochemical Co. Ltd.

Different compositions of Chs\HA mixtures were prepared by dissolving ethanol sterilized Chs and HA in deionized water. Type I collagen into deionized water at room temperature until completely dissolved. Chs\HA mixtures were blended with Col solution at different weight ratios to form a bioink, which has a suitable viscosity to ensure 3D bioprinting. To improve the mechanical strength of tissue engineered scaffolds, Tyrosineparticle was dispersed in the polymer solution. The boinks composed of Chs/Col/HA, and different amount of Tyrosine were labeled as shown in Table1.

Table1. Compositions of bioinks

\begin{tabular}{ll}
\hline $\mathrm{S}$ & Composition \\
\hline 1 & $8 \%$ Chs- $0.5 \%$ HA-10\% Col \\
2 & $8 \%$ Chs- $0.5 \%$ HA- $-10 \%$ Col- $3 \%$ Tyrosine \\
3 & $8 \%$ Chs- $0.5 \%$ HA- $10 \%$ Col-6\% Tyrosine \\
4 & $8 \%$ Chs- $0.5 \%$ HA-10\% Col-9\% Tyrosine \\
\hline
\end{tabular}

3D tissue engineered skin substitutes were fabricated using a computer aided 3D bioplotter (EnvisionTEC, Germany). The schematic of the 3D bioprinting process is shown in Figure 1a. The dimension of 3D printed structure was $10 \mathrm{~mm}$ x $10 \mathrm{~mm} \times 1.2$ mm made of 6 layers as shown in Figure 1b. Each layer was deposited at an angle of $90^{\circ}$ to the underlying layer as shown in Figure 1c. Meantime, 10wt \% STPP solution was sprayed onto the struts for 15 min after printing each layer. Printing speed was adjusted according to the viscosity of the bioinks. The cross-linked skin substitutes then were immersed into PBS overnight before cell culture.

The tensile properties of the skin substitutes made of bioinks were evaluated using a universal testing machine (HY-0230, China) at the speed of $5 \mathrm{~mm} / \mathrm{min}$. The skin substitute samples were printed following the standard test specimen and the tensile stress-strain curve was obtained.In vitro degradation of tissue engineered skin substitutes was evaluated for 28 days with the addition of lysozyme(Sigma, powder, $70000 \mathrm{U} / \mathrm{mg}$ ) from chicken egg white. The percentage weight loss of the tissue engineered skin substitutes was determined by the formula (1): 
$\mathrm{W}=(\mathrm{Wi}-\mathrm{Wt}) / \mathrm{Wi}$.

Where $\mathrm{W}_{\mathrm{i}}$ is the initial dry weight of the scaffolds and $\mathrm{W}_{\mathrm{t}}$ is the final weight after 0 , $1,4,7,14$ and 28 days of incubation. The degradable ratio was determined for each scaffolds. The experiments was performed in triplicates.

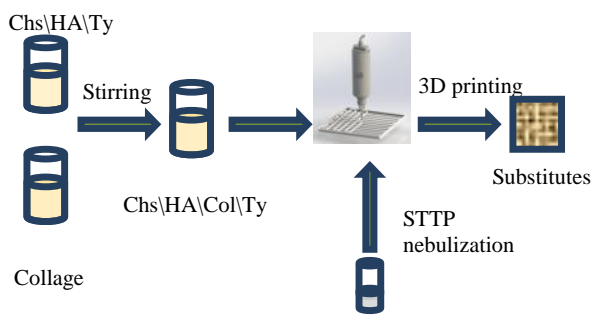

(a)

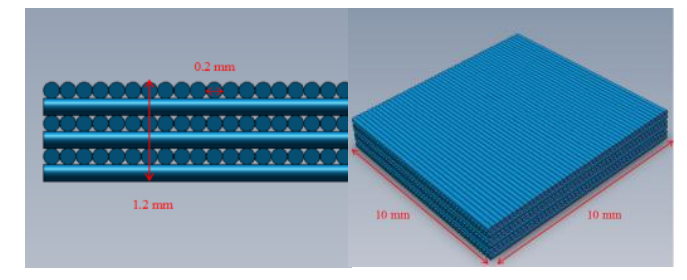

(b)

(c)

Figure 1. 3D bioprinting process (a) fabrication process (b) layout of skin substitutes (c) cross-section of the skin substitutes

HaCat keratinocytes (P4, purchased from ScienCell Research Laboratories, China) were maintained in a humidified $5 \% \mathrm{CO} 2$-containing atmosphere $\left(37^{\circ} \mathrm{C}\right)$. Cell viability test of the skin substitutes at days 1, 4, 7 was conducted using a live/dead kit (LIVE/DEAD® Viability/Cytotoxicity Kit, Invtrogen, USA). The skin substitutes were pre-sterilized and were immersed in DMEM for two hours before cell seeding. Cellsof $5 \times 104$ were seeded on each skin substitutes. Then, skin substitutes were incubated for $24 \mathrm{~h}$ in a humidified $5 \% \mathrm{CO} 2$-containing atmosphere $\left(37^{\circ} \mathrm{C}\right)$.

\section{Results and Discussion}

Both skin substitutes made of sample 1 and sample 3 exhibited human skin features in terms of color and elasticity as shown in Figure 2(a) and (b). It can be seenthat the surfaces of skin substitutes made of Sample 1 and Sample 3 has no significant difference. The micro-particles of Tyrosine can be clearly seen in Figure2(d). The layered structure by 3D printing can be seen in Figure 2(e) and (f). It also shows that the bioinks developed in this paper is suitable for fabrication stratified skin substitutes.

Mechanical properties such as tensile stress and elongation at break of different skin substitutes were measured and presented in Figure 3. Tensile strength of Sample 1 and Sample 3 were recorded as 29.9 and $39.1 \mathrm{kPa}$, respectively. The tensile stress was close to the human skin ranged from 2.16 to $107 \mathrm{kPa}[16]$. Elongation at break of Sample 1 and Sample 3 were recorded as $63.5 \%$ and $52.2 \%$, respectively as shown in Figure 3(a).

The degradation degrees of sample 1 and 3 in 14 days were $39 \%$ and $31 \%$, respectively, as shown in Figure 3(b). It indicated that Tyrosine could speed up the degradation rate of skin substitutes. 


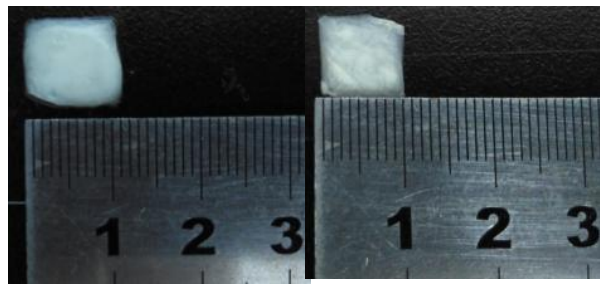

(a)

(b)

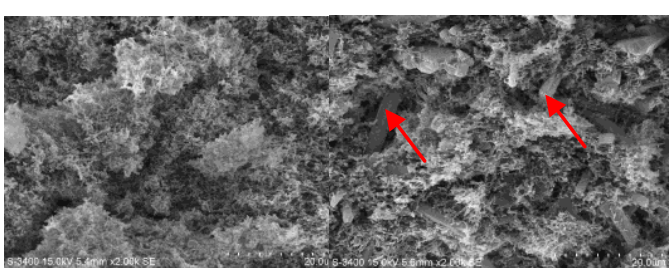

(c)

(d)

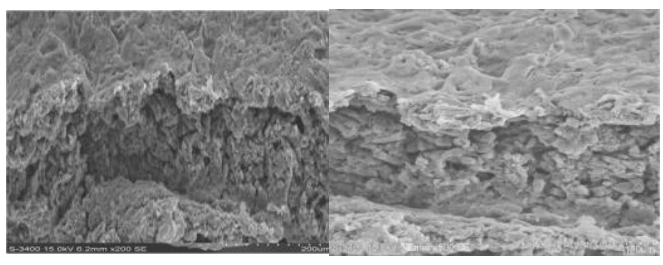

(e)

(f)

Figure 2. Morphologies of the skin substitutes (a) (c) (e) SEM photos of Sample 1; (b) (d) (e) SEM photos of Sample 3 (Arrows indicate the Tyrosine particles)
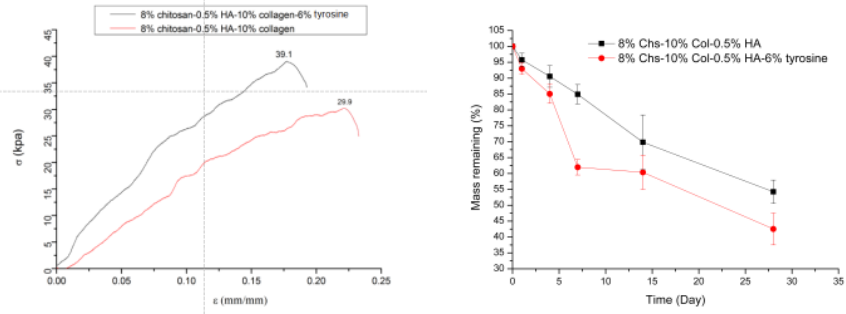

Figure 3. Physical characteristics. (a) Tensile stress-strain curve; (b) Degradation test

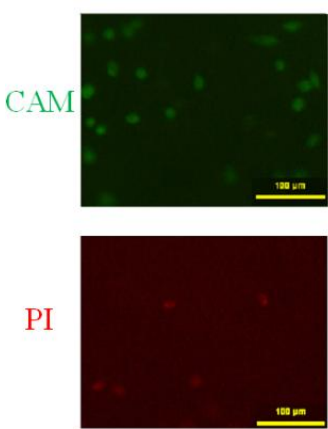

(a)
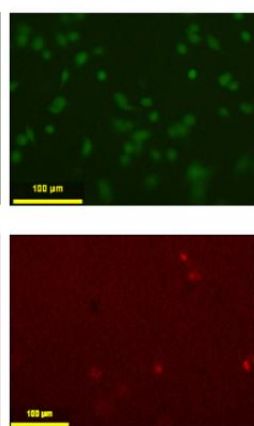

(b)
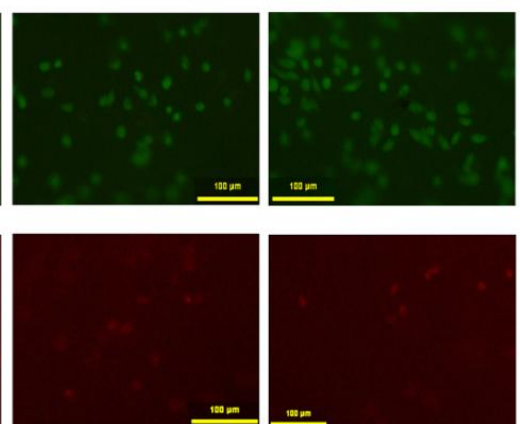

(c)

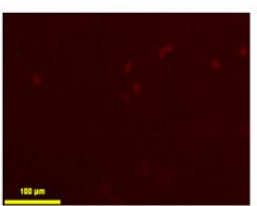

(d)
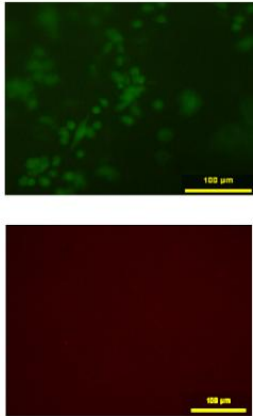

(e)

Figure 4. The Cam/PI staining of HaCat cells on different skin substitutes after 3 days: (a) Sample 1; (b) Sample 2; (c) Sample 3; (d) Sample 4; (e) Control group 
Cell morphology of HaCat cells was shown in Figure 4, which indicated that Sample 1 and 3 skin substitutes were maintained high viability and no significant changes in cell viability were observed in sample 1, sample 2, sample 3 and sample 4 after culturing 3 days. Moreover, as shown in the Figure 5, no significance was fund in cell viability in the skin substitutes compared to the control group, which indicated that the Tyrosine particles didn't effect cell viability.

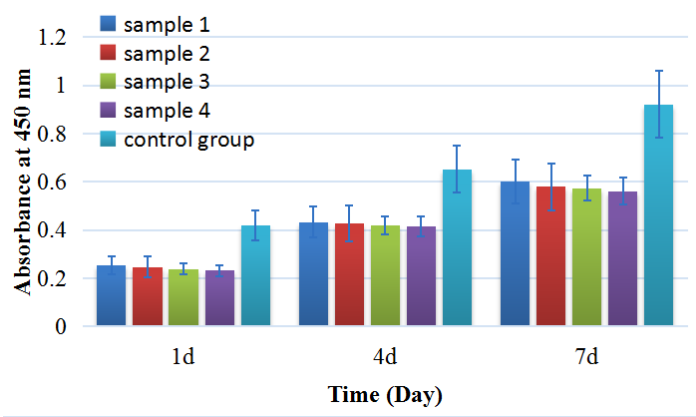

Figure. 5. Hacat cells growth on different materials (The $\mathrm{P}>0.05$ )

\section{Conclusion}

A novel design of bioinks by combining multi-natural biomaterials with Tyrosine particle reinforcement was developed. Skin scaffolds were fabricated by 3D printing using the designed bioinks. The morphology, mechanical, thermal and degradation tests showed that the skin substitutes made of the bioinks were mechanically strong and able to form predefined structure. Bioinks developed in this paper are suitable for fabrication of cell-laden skin substitutes.

\section{Acknowledgements}

The authors would like to thank the following grant agencies or sponsors for making this research possible. The first is the Opening Project of Shanghai Key Laboratory of Orthopedic Implant (KFKT2016001), the second is the 1000 Talents Program.

\section{References}

[1] Zhong S.P., Zhang Y.Z. and Lim C.T. Tissue scaffolds for skin wound healing and dermal reconstruction. Wiley Interdisciplinary Reviews Nanomedicine \& Nanobiotechnology, 2, 2010, 510-525.

[2] Wang X., You C., Hu X., Zheng Y., Li Q. , FengZ., Sun H. , Gao C., Chun H., The roles of knitted mesh-reinforced collagen-chitosan hybrid scaffoldin the one-step repair of full-thickness skin defects in rats. ActaBiomaterialia, 9, 2013, 7822-7832.

[3] Monteiro I.P., Gabriel D., Timko B.P., Hashimoto M., Karajanagi S., Tong R., Marques A.P., Reis R.L., Kohane D.S. A two-component pre-seeded dermal-epidermal scaffold. ActaBiomaterialia 10, 2014, 4928-4938. 
[4] Casale C., ImparatoG., UrciuoloF., Netti P.A. Endogenous human skin equivalent promotes in vitro morphogenesis of follicle-like structures. Biomaterials, 101, 2016, 86-95.

[5] O'Brien C.M., Holmes B., Faucett S., Zhang L.G. Three-dimensional printing of nanomaterial scaffolds for complex tissue regeneration. Tissue Engineering Part B Reviews. 2015, 21(1):103-14.

[6] Lee W., Debasitis J.C., Lee V.K., Lee J.H., Fischer K., Edminster K., Park J.K., Yoo S.S. Multi-layered culture of human skin fibroblasts and keratinocytes through three-dimensional freeform fabrication. Biomaterials. 2009, 30(8):1587-1595.

[7] Huang S.,Yao B.,Xie J. 3D bioprinted extracellular matrix mimics facilitate directed differentiation of epithelial progenitors for sweat gland regeneration. ActaBiomaterialia,9,2015,32:170-177.

[8] Zhang H.B,,Xing T.L., Shi Y., Yin R.X., Yang S.M.and Zhang W.J. 3D bioprinting is not only about cell-laden structures. Chinese Journal of Traumatology, $19,2016,187-192$.

[9] Pereira R.F.,Bártolo P.J. 3D bioprinting of photocrosslinkable hydrogel constructs, Journal of Applied Polymer Science. 132,2015, 42458.

[10] Visser J., Melchels F.P., Jeon J.E., Van Bussel E.M., Kimpton L.S., Byrne H.M., Dhert W.J.A., Dalton P.D., Hutmacher D.W. andMalda J. Reinforcement of hydrogels using three-dimensionally printed microfibres. Nature Communications. 2015, 6.

[11]Tsai YC, Li S, Hu SG, Chang WC., Jeng US., Hsu SH. Synthesis of ThermoresponsiveAmphiphilic Polyurethane Gel as a New Cell Printing Material near Body Temperature. Acs Applied Materials \& Interfaces.2015: 27613-27623.

[12]Hong S., Sycks D., Chan H.F., Lin S.T., Lopez G.P., Guilak F., Leong K.W., Zhao X.H. 3D Printing of Highly Stretchable and Tough Hydrogels into Complex, Cellularized Structures. Advanced Materials. 2015, 27(27):4035-4040.

[13] Shin S.R., Bae H., Cha J.M., Mun J,Y., Chen Y,C., Tekin H., Shin H., Farshchi S., Dokmeci M.R. Carbon Nanotube Reinforced Hybrid Microgels as Scaffold Materials for Cell Encapsulation. AcsNano. 2012, 6(1):362-72.

[14]Baldea I., Mocan T., Cosgarea R., The role of ultraviolet radiation and tyrosine stimulated melanogenesis in the induction of oxidative stress alterations in fair skin melanocytes, Experimental Oncology, 2009,31(4):200-208

[15] Kilfoyle B.E., Sheihet L., Zhang Z., Laohoo M., Kohn J., Michniak-kohn B.B., Development of paclitaxel-TyroSpheres for topical skin treatment, Journal of Controlled Release, 163(1), 2012, 18-24.

[16]Fazekas I G, Kósa F, Basch A. [On the effect of constitutional factors (body height) on the tensile strength of the human skin. GegenbaursMorphologischesJahrbuch, 1969, 113(113):295-302. 